\title{
The role of mTOR signaling pathway on cognitive functions in cerebral ischemia-reperfusion
}

\author{
LIYING YANG $^{1 *}$, YINGYING ZHANG ${ }^{1 *}$, ZHONGRUI YAN $^{2}$ and FENG TIAN ${ }^{2}$ \\ ${ }^{1}$ Department of Neurology, The Affiliated Hospital of Qingdao University, Qingdao, Shandong 266003; \\ ${ }^{2}$ Department of Neurology, Jining No. 1 People's Hospital, Jining, Shandong 272000, P.R. China
}

Received October 17, 2016; Accepted April 18, 2017

DOI: 10.3892/etm.2017.4881

\begin{abstract}
The role and mechanism of the mTOR signaling pathway in the impaired cognitive function in cerebral ischemia-reperfusion were examined in the present study. Sprague-Dawley (SD) rats were divided into the sham operation, cerebral ischemia, cerebral ischemia-reperfusion and cerebral ischemia-reperfusion adaptive groups. A Morris water maze test was carried out in the different treatment groups at 2 weeks after surgery to detect cognitive function. After the experimental animals were sacrificed, fluorescent quantitative PCR test was used to detect the key signaling molecules in the mTOR signaling pathway in the different treatment groups, such as mTOR, p-mTOR, AKT and p-AKT gene mRNA expression. The protein expression was determined by enzyme-linked immunosorbent assay and western blotting. mTOR expression and localization in the different treatment groups was detected by immunohistochemistry, and the positive cell rate was determined. Compared with the sham operation group, the levels of mTOR, p-mTOR, AKT and p-AKT mRNAs and hippocampal proteins were significantly lower in the cerebral ischemia group and cerebral ischemia-reperfusion group $(\mathrm{P}<0.05)$. Levels of mTOR, $\mathrm{p}-\mathrm{mTOR}, \mathrm{AKT}$ and $\mathrm{p}-\mathrm{AKT}$ mRNAs and proteins in the cerebral ischemia-reperfusion adaptive group decreased but did not show significant differences $(\mathrm{P}>0.05)$. The Morris water maze results showed that, the adaptive ability and the cognitive functions were improved significantly in the cerebral ischemia-reperfusion adaptive group when compared with the cerebral ischemia and cerebral ischemia-reperfusion groups $(\mathrm{P}<0.05)$. The number of mTOR-positive cells in hippocampus was significantly higher in the sham operation
\end{abstract}

Correspondence to: Dr Feng Tian, Department of Neurology, Jining No. 1 People's Hospital, 6 Jiankang Road, Jining, Shandong 272000, P.R. China

E-mail:wgcv_4255@163.com

*Contributed equally

Key words: mTOR, signal pathway, cerebral ischemia-reperfusion, cognitive function and cerebral ischemia-reperfusion adaptive groups, but there was no difference between these groups. In conclusion, mTOR signaling pathway improves the cognitive function in cerebral ischemia-reperfusion in rats.

\section{Introduction}

Cerebral circulation disorders such as the incidence of sudden ischemic cerebral events have been on the increase in China (1). Statistical results show that, there were approximately 800,000 individuals suffering from ischemic cerebral disease in China in 2015 (2). Due to its rapid onset and high mortality rate, ischemic cerebral disease has become the third leading cause of death and primary disabling disease. Clinical statistics show that, cerebral ischemic reperfusion often leads to different degrees of brain injury, accompanied by cognitive dysfunction, mental decline, epilepsy and other diseases (3). These diseases seriously adversely affect patient health and quality of life. Therefore, the mechanisms of reperfusion after cerebral injury has become an important field in the study of cerebral ischemia-reperfusion (4).

Previous findings suggested that as a serine/threonine protein kinase, mTOR signaling pathway plays an important role in cell growth, proliferation, apoptosis and other physiological processes (5). The mTOR signaling pathway is associated with various neurological diseases, such as epilepsy, Alzheimer's disease and many other cognitive dysfunction diseases (6-8). In addition, the enhancing activity of key signaling molecules in mTOR signaling pathway such as AKT, can inhibit programmed cell death of neurons $(6,7)$ promoting improvement of cognitive function. The mTOR signaling pathway is composed of a plurality of genes, and is associated with numerous signal transduction pathways, such as the PI3K/AKT signal path (8). AKT gene promotes cell proliferation and metabolism, and can inhibit cell death to a certain extent. Additionally, AKT promoted the increase of NO levels in cells, thereby reducing the incidence of vasospasm and promoting the dissolution of thrombus (9). Thrombosis is thought to be an important inducing factor of other related diseases following cerebral ischemia-reperfusion.

To the best of our knowledge, this is the first study to investigate the role and mechanism of mTOR signaling pathway in the impaired cognitive function in cerebral ischemia-reperfusion to reveal the correlation between mTOR signaling 
pathway and the cognitive dysfunction in cerebral ischemiareperfusion, and provide some theoretical and experimental basis for the treatment of cerebral ischemia.

\section{Materials and methods}

\section{Materials}

Experimental animals. The present study was conducted on 48 Sprague-Dawley (SD) rats (average body weight, $248 \pm 37 \mathrm{~g}$; age, $2.4 \pm 0.6$ weeks). The rats were divided into the sham operation, cerebral ischemia, cerebral ischemia-reperfusion and the cerebral ischemia-reperfusion adaptive groups (addition of $0.5 \mathrm{mmol}$ anthocyanin when reperfusion).

Main reagents. The reagents used were RNA kit (Takara Bio, Dalian, China). mTOR, p-mTOR, AKT and p-AKT primary antibodies were goat anti-rabbit antibodies and rabbit anti-rat secondary antibodies labeled by HRP (Acris Antibodies, San Diego, CA, USA). Animal cells total protein extraction kit (Axygen Biosciences, Union City, CA, USA) and immunohistochemistry kit (Tiangen Biotech Co., Ltd., Beijing, China) were also used.

Instruments. Fluorescence quantitative PCR instrument (Applied Biosystems, Foster City, CA, USA); gel imaging instrument (Bio-Rad Laboratories, Inc., Hercules, CA, USA); low temperature refrigerated centrifuge (Hitachi, Tokyo, Japan); ultra low temperature freezer (Haier, Beijing, China); and protein electrophoresis apparatus (Beijing Liuyi Biotechnology Co., Ltd., Beijing, China) were used in the study.

\section{Methods}

Morris water maze test. The water maze test used in this study was carried out as described by Porcile et al (10).

Step-down test. The step-down test in this study was performed according to Lu et al (11).

\section{Fluorescence quantitative PCR}

$R N A$ extraction. The hippocampal tissue samples were used as the research object. Equal volume splitting solution was added in a cold EP tube. RNA Plus was also added after percussion uniformly with transfer liquid gun and the specific instructions of the experimental protocol were followed.

Fluorescence quantitative PCR. In order to detect the RNA expression of mTOR, p-mTOR, AKT and p-AKT in different samples, we used SYBR-Green 1 dye method, performed as per specific experimental protocol instructions. Primers used were produced by Suzhou Genewiz Biological Engineering Co., Ltd. (Suzhou, China) (Table I).

Enzyme-linked immunosorbent assay (ELISA). The experiment was carried out according to the specification of ELISA kit (Axygen Biosciences) with improvements (12). Total protein was extracted and quantified with Coomassie Brilliant Blue staining. The total protein of $100 \mathrm{mg}$ was diluted by diluent in the kit. Then, $100 \mu \mathrm{l}$ was added to $50 \mu \mathrm{l}$ test solution for ELISA test to measure the expression of different proteins.

Western blotting. In this study, we used an animal cells total protein extraction kit (Axygen Biosciences) to extract total protein from samples. The specific instructions of the
Table I. Primers for fluorescence quantitative PCR.

\begin{tabular}{ll}
\hline Molecules & \multicolumn{1}{c}{ Primer sequences } \\
\hline mTOR-F & GTAGCTAGCTAGCATCGTCCAG \\
mTOR-R & GTCGTAGCTGCTAGCTACGTCG \\
p-mTOR-F & CGTAGGGATGCTATGATTCGC \\
p-mTOR-R & GTAGTTAGTCGCGTAGCTGACTC \\
AKT-F & GCTGGCTGATGCTAGCTACCG \\
AKT-R & GTGTCGGCAGTCGTAGCAGC \\
p-AKT-F & GTGAGCAGTGCTGACGCTACG \\
p-AKT-R & GTAGGCTAGCCAGTCGATGCTCG \\
GAPDH-F & CGTAGGGCTAGCTAGCTAGATAC \\
GAPDH-R & CGTAGCTGAGAGTTAGCTAGCATC
\end{tabular}

F, forward; R, reverse.

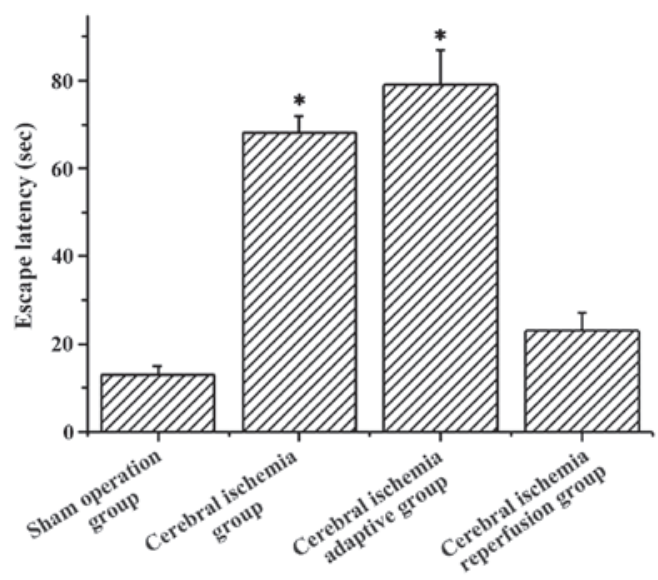

Figure 1. Results of water maze test. ${ }^{*} \mathrm{P}<0.05$ indicates significant difference between groups.

protocol were referred to with improvements (13). Coomassie Brilliant Blue staining was used to quantify after extracting total protein. Rabbit polyclonal mTOR antibody (dilution, 1:500; cat. no. ab2732), rabbit monoclonal p-mTOR antibody (dilution, 1:500; cat. no. ab109268), rabbit polyclonal AKT antibody (dilution, 1:500; cat. no. ab8805) and rabbit monoclonal p-AKT antibody (dilution, 1:500; cat. no. ab81283) were all purchased from Abcam (Cambridge, MA, USA).

Immunohistochemistry. In this study, routine antibody incubation and staining of tissue samples from mouse hippocampal CA3 region were performed with streptavidin-peroxidase (SP) method. The criteria for immunohistochemical evaluation were as follows: Membrane staining ratio $<10 \%$ or negative after staining was considered as negative; only the cell membrane stained or membrane staining ratio $>10 \%$ was considered positive.

Statistical analysis. Data are expressed as mean \pm standard deviation. Statistical analysis was performed using SPSS 10.0 software (Chicago, IL, USA). P $<0.05$ was considered to indicate a statistically significant difference. 


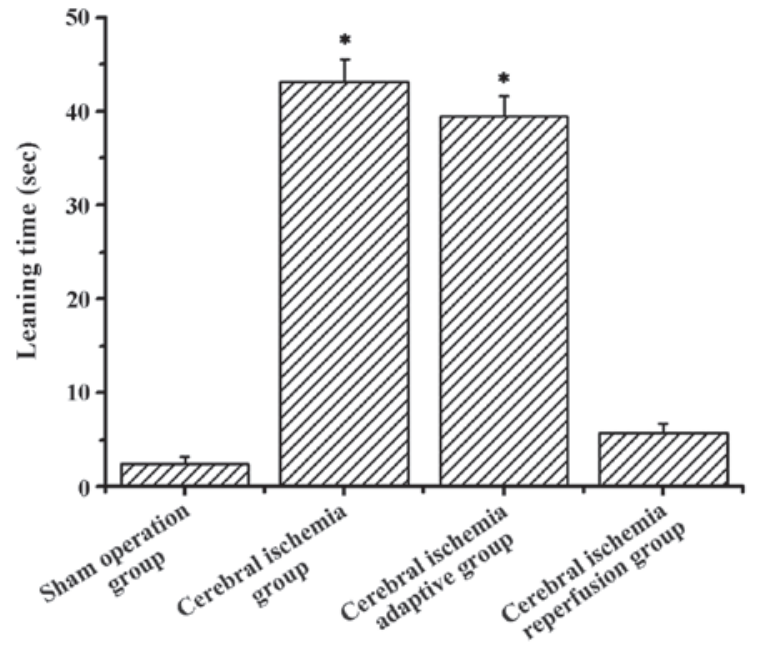

Figure 2. Results of step-down test. " $\mathrm{P}<0.05$ indicates significant difference between groups.

\section{Results}

Morris water maze test in rats. Water maze test results showed that (Fig. 1) the escape latency in the cerebral ischemia group and the cerebral ischemia-reperfusion group were 69.3 and $75.3 \mathrm{sec}$, respectively, which differed significantly from that in the sham operation group $(11.2 \mathrm{sec})(\mathrm{P}<0.05)$. It suggested that cerebral ischemia and subsequent reperfusion affected the memory ability of rats to a certain extent.

Step-down test in rats. Step-down test results showed that (Fig. 2) in comparison with the sham operation group (7.6 sec), learning time was greatly extended in the cerebral ischemia group (43.2 sec) and the cerebral ischemia-reperfusion group $(39.7 \mathrm{sec})$ or the learning ability of rats decreased significantly in the latter two groups $(\mathrm{P}<0.05)$. Although the learning ability of the cerebral ischemia-reperfusion adaptive group $(11.2 \mathrm{sec})$ was poorer than that of the sham operation group, there was no significant difference between the two groups $(\mathrm{P}>0.05)$. The results indicated that cerebral ischemia and subsequent reperfusion affected the learning ability of rats to a certain extent.

Detection of mRNA gene in rats of the different treatment groups. In this experiment, rat hippocampus tissue samples in the treatment groups were investigated. Expression of mTOR, p-mTOR, AKT and p-AKT mRNAs in different samples were measured by fluorescence quantitative PCR. The results are shown in Fig. 3. Levels of mTOR, p-mTOR, AKT and p-AKT mRNAs in hippocampus in rats of the sham operation group were, respectively 13.2-, 23.6-, 14.3- and 28.5-fold than that in the cerebral ischemia group, and there were significant
A

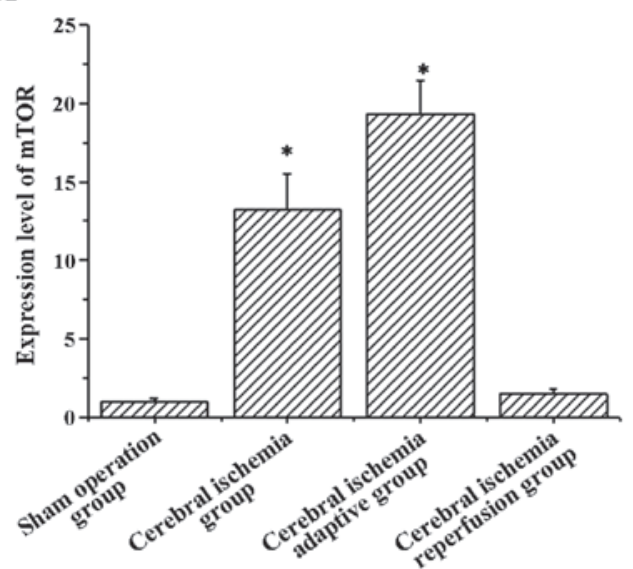

C

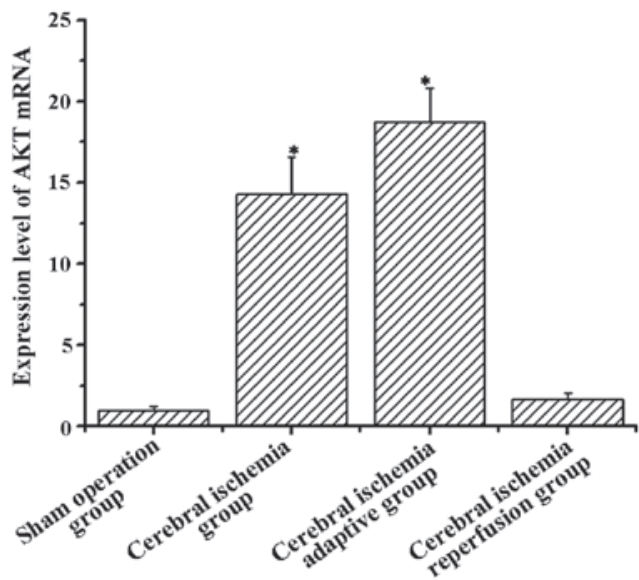

B

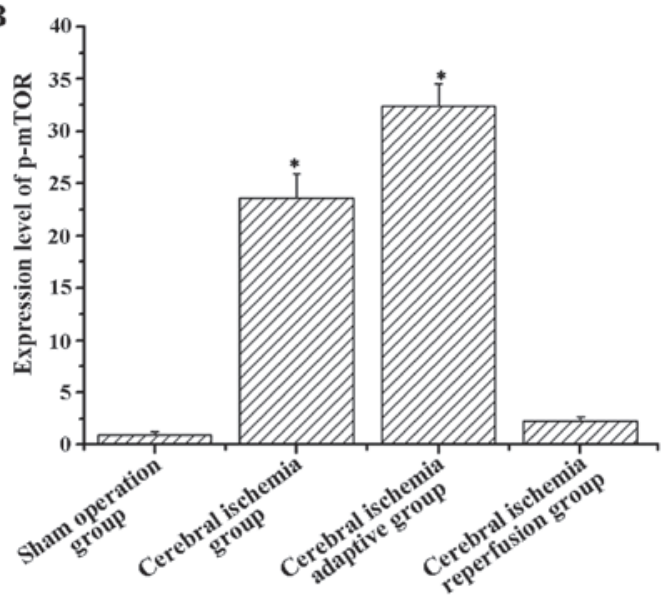

D

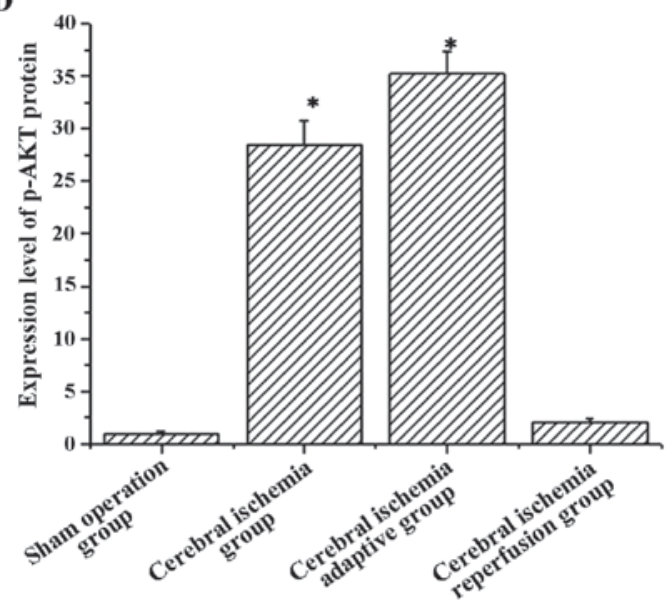

Figure 3. Expression of different mRNAs in rats of different treatment groups. Expression level of (A) mTOR mRNA, (B) p-mTOR mRNA, (C) AKT mRNA, and (D) p-AKT mRNA. "P<0.05 indicates a significant difference between groups. 

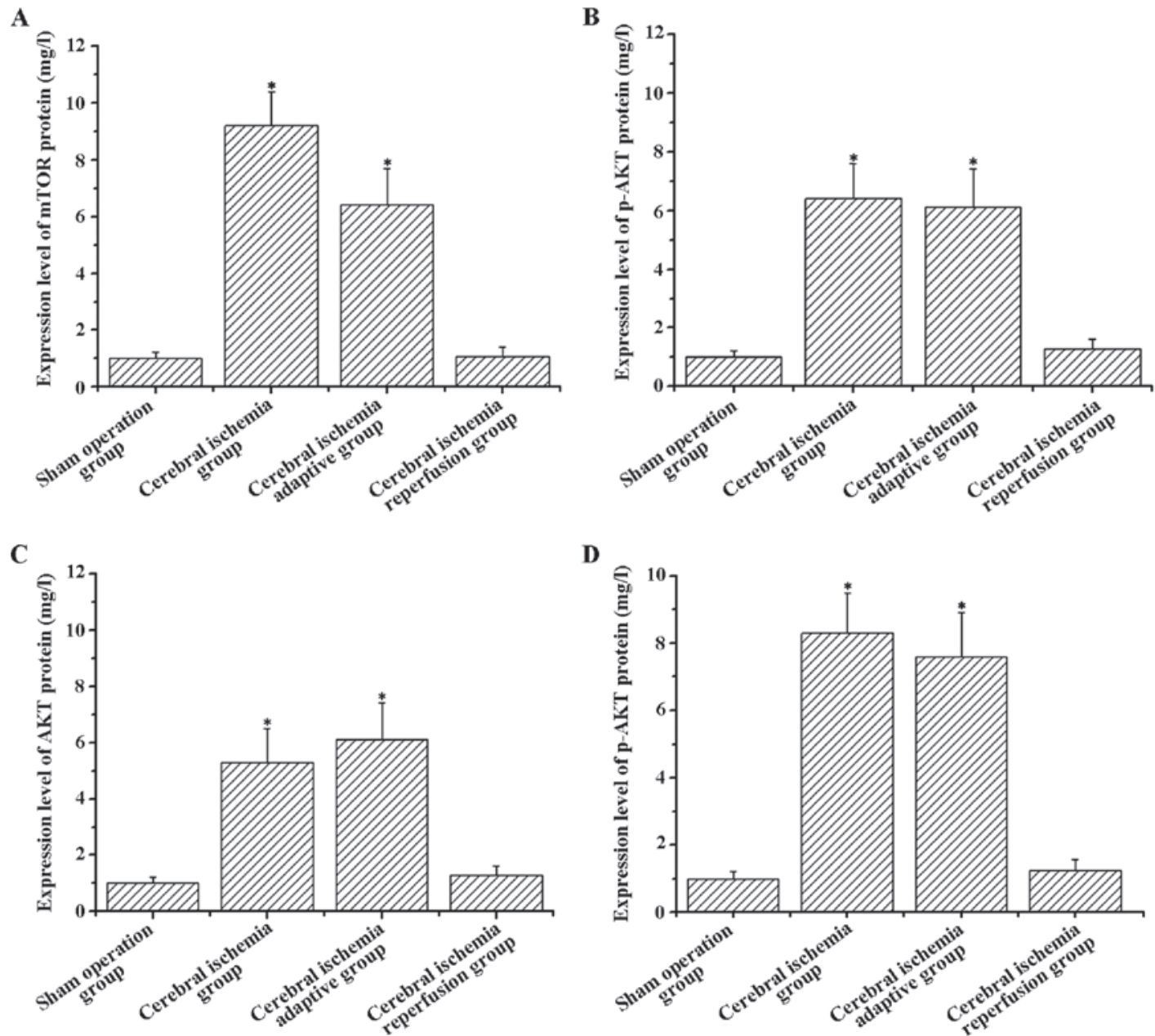

Figure 4. Expression of different proteins in rats of different treatment groups. Expression level of (A) mTOR protein, (B) p-mTOR protein, (C) AKT protein, and (D) p-AKT protein. ${ }^{*} \mathrm{P}<0.05$ indicates a significant difference between groups.

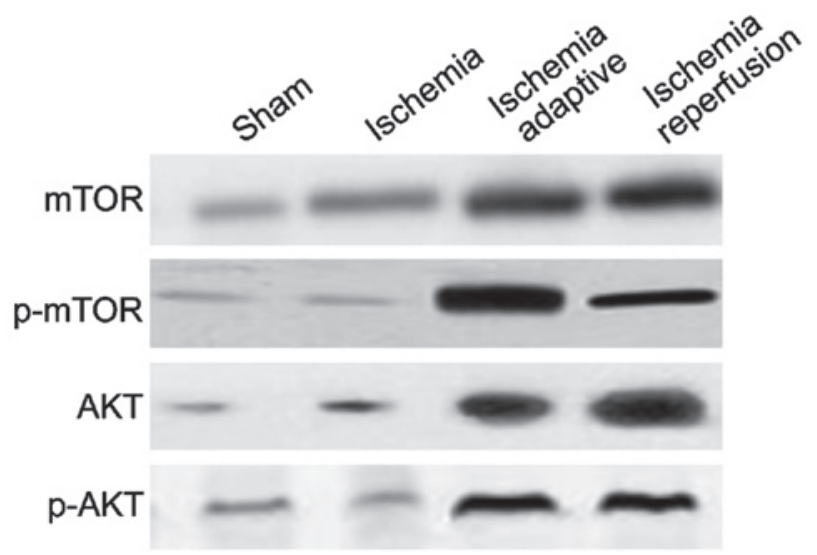

Figure 5. Detection of protein levels in rats of the different treatment groups by western blotting.

differences in each mRNA level between the two groups $(\mathrm{P}<0.05)$. Levels of the above mRNAs in the cerebral ischemiareperfusion adaptive group were, respectively, 19.3-, 32.4-, 18.7- and 35.2-fold that in the cerebral ischemia group, and there were significant differences in each mRNA level between the groups $(\mathrm{P}<0.05)$. The levels of mTOR, p-mTOR, AKT and p-AKT mRNAs in the cerebral ischemia-reperfusion group were, respectively, 1.5-, 2-, 3-, 1.7- and 2.1-fold that in the cerebral ischemia group, and there were no significant differences $(P>0.05)$. This indicated that there was a correlation between mTOR signaling pathway and cerebral ischemia-reperfusion.

Detection of proteins in rats of the different treatment groups. We investigated proteins extracted from different samples. The levels of mTOR, p-mTOR, AKT and p-AK proteins in the different groups were determined by ELISA. The results are shown in Fig. 4. Expression of mTOR protein in the sham operation group was significantly higher than that in the cerebral ischemia and cerebral ischemia-reperfusion groups $(\mathrm{P}<0.05)$. By contrast, the mTOR protein level did not differ significantly between the cerebral ischemia-reperfusion adaptive and sham operation groups $(\mathrm{P}>0.05)$. $\mathrm{p}-\mathrm{mTOR}$, AKT and $\mathrm{p}$-AKT protein levels all showed the same trend.

Detection of protein levels in rats of different treatment groups by western blotting. The levels of mTOR, p-mTOR, AKT and p-AKT proteins in different groups were determined by western blotting. The results are shown in Fig. 5 . As seen in Fig. 5, levels of mTOR, p-mTOR, AKT and p-AKT proteins in the sham operation group and cerebral ischemia-reperfusion adaptive group were significantly higher than in the cerebral ischemia group and cerebral 


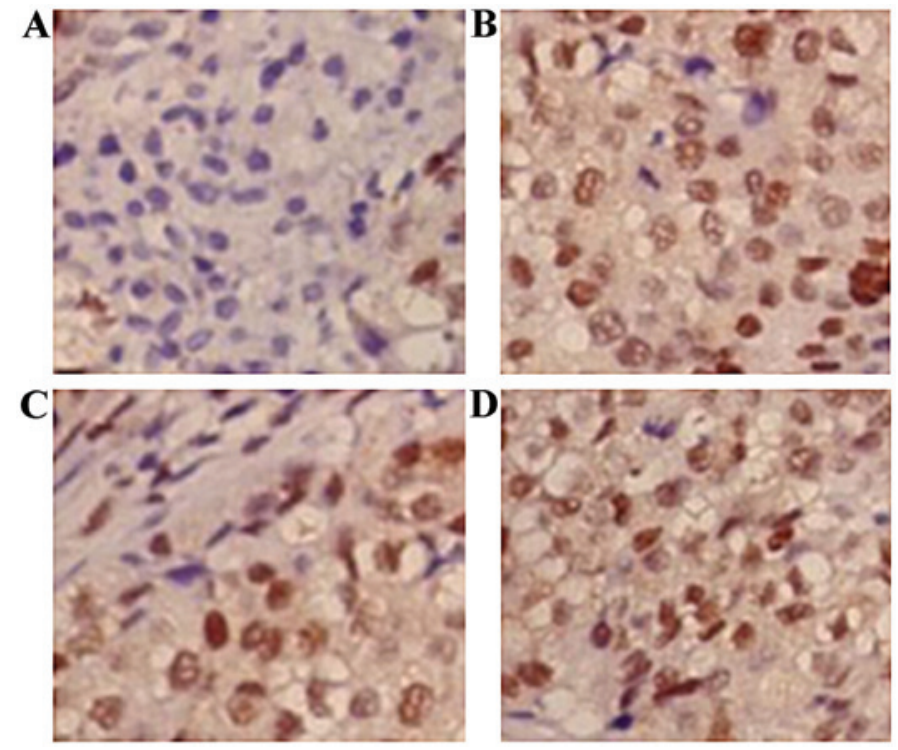

Figure 6. Immunohistochemical detection in rats of the treatment groups. (A) Sham operation group. (B) Cerebral ischemia group. (C) Cerebral ischemia-reperfusion adaptive group. (D) Cerebral ischemia-reperfusion group. Magnifiication, x100.

Table II. The number of immunohistochemically positive cells in rats of the different treatment groups.

\begin{tabular}{lccccr}
\hline Groups & $\begin{array}{c}\text { Cell } \\
\text { counts }\end{array}$ & $\begin{array}{c}\text { Positive cell } \\
\text { counts (N) }\end{array}$ & $\begin{array}{c}\text { Positive cell } \\
\text { rate }(\%)\end{array}$ & $\begin{array}{c}\text { Negative cell } \\
\text { counts (N) }\end{array}$ & $\begin{array}{c}\text { Negative cell } \\
\text { rate }(\%)\end{array}$ \\
\hline Sham operation & 400 & 376 & 94 & 24 & 6 \\
Cerebral ischemia & 400 & 36 & 9 & 364 & 91 \\
Cerebral ischemia-reperfusion adaptive & 400 & 328 & 82 & 72 & 18 \\
Cerebral ischemia-reperfusion & 400 & 42 & 10.5 & 358 & 89.5 \\
\hline
\end{tabular}

ischemia-reperfusion group, which was consistent with the ELISA results.

Immunohistochemical detection in rats of the different treatment groups. The distribution and expression of mTOR protein in the different treatment groups were detected by immunohistochemistry. As shown in Fig. 6 when compared with the sham operation and cerebral ischemia-reperfusion adaptive groups, the expression of mTOR protein in the hippocampus of rats was lower in the cerebral ischemia group and cerebral ischemia-reperfusion group, which was consistent with the ELISA results. The mTOR-positive cell count results in the different treatment groups (Table II) showed that the numbers of mTOR-positive cells in the hippocampus of rats in the sham operation group (84.3\%) and cerebral ischemia-reperfusion adaptive group $(65.3 \%)$ were significantly higher than that in the cerebral ischemia group $(9.03 \%)$ and cerebral ischemia-reperfusion group (7.54\%). This suggested that cerebral ischemia and cerebral ischemia-reperfusion reduced the mTOR protein level in the hippocampus.

\section{Discussion}

Clinical statistics show that, cerebral ischemia, is a common disease in modern society, having high morbidity, disability and mortality rate, and is one of the main brain diseases endangering the lives of patietns (13). Previous findings have shown that the main treatment method for cerebral ischemia is the timely recovery of blood perfusion in the ischemic area of the brain (14). There is evidence from clinical results indicating that cerebral reperfusion does not promote the recovery of brain hypoxia (15). Instead it leads to cerebral ischemic tissue dysfunction resulting in further aggravation in the patient's condition (16). An animal study on cerebral ischemia in rats showed that the cognitive ability decreased after cerebral ischemia-reperfusion $(17,18)$. The detection results in the hippocampus showed that the neurons in the CA1 area were severely damaged, and the learning and cognitive ability decreased. This suggests inability of brain ischemic reperfusion to restore neurons, further causing structural damage to neurons to a large extent (19), however, the mechanism involved is not clear. It has been shown that the addition of hydrogen salt solution when reperfusion improved the injury of cerebral neurons by cerebral ischemia-reperfusion to a certain extent, which was characterized by the result that there was no significant decrease in cognitive function in rats (20). The addition of anthocyanin during reperfusion also alleviates the damage in cognitive function to some extent, but the signal transduction pathways involved are not clear (21).

In the present study, we investigated the mTOR signaling pathway in the hippocampus of rats before and after cerebral ischemia-reperfusion. The results showed that, mTOR 
signaling pathway related signaling molecules and gene expression significantly decreased in the cerebral ischemia and cerebral ischemia-reperfusion groups when compared with the healthy rats $(\mathrm{P}<0.05)$. Moreover, in rats with appropriate treatment after cerebral ischemia-reperfusion, there was no significant difference in the expression of mTOR signaling pathway related genes in hippocampus when compared with healthy rats $(\mathrm{P}>0.05)$, and its cognitive function was not significantly decreased, suggesting the decline of cognitive function in rats after cerebral ischemia-reperfusion may be generated through the mTOR signaling pathway. Further studies on the mTOR signaling pathway have found that the mTOR signaling pathway participates in many physiological and biochemical reactions in cells $(22,23)$. For example, previous results have shown that the mTOR signaling pathway can sense the changes of intracellular energy as well as intracellular nutrients, thus regulating many physiological reactions in cells including gene transcription, translation, ribosome generation and amino acid intake. Furthermore, the mTOR signaling pathway plays an important role in the regulation of multiple neural processes, such as formation and development of the brain, formation and maturation of synapses and neural cell proliferation $(22,23)$.

In this study, it is clarified that mTOR signaling pathway plays a role of regulation in cognitive dysfunction after cerebral ischemia-reperfusion, which provides some theoretical and experimental basis for the treatment of cerebral ischemia.

\section{References}

1. Wang S, Chong ZZ, Shang YC and Maiese K: Wnt1 inducible signaling pathway protein 1 (WISP1) blocks neurodegeneration through phosphoinositide 3 kinase/Akt1 and apoptotic mitochondrial signaling involving Bad, Bax, Bim, and Bcl-xL. Curr Neurovasc Res 9: 20-31, 2012.

2. Chong ZZ, Shang YC, Wang S and Maiese K: SIRT1: new avenues of discovery for disorders of oxidative stress. Expert Opin Ther Targets 16: 167-178, 2012.

3. Xie Z, Chen F, Wu X, Zhuang C, Zhu J, Wang J, Ji H, Wang Y and Hua X: Effects of supplemental erythropoietin on its receptor expression and signal transduction pathways in rat model of retinal detachment. Curr Eye Res 37: 138-144, 2012.

4. Yu Y, Ren QG, Zhang ZH, Zhou K, Yu ZY, Luo X and Wang W: Phospho-Rb mediating cell cycle reentry induces early apoptosis following oxygen-glucose deprivation in rat cortical neurons. Neurochem Res 37: 503-511, 2012.

5. Pineda D, Ampurdanés C, Medina MG, Serratosa J, Tusell JM, Saura J, Planas AM and Navarro P: Tissue plasminogen activator induces microglial inflammation via a noncatalytic molecular mechanism involving activation of mitogen-activated protein kinases and Akt signaling pathways and AnnexinA2 and Galectin-1 receptors. Glia 60: 526-540, 2012.

6. Shang YC, Chong ZZ, Wang S and Maiese K: Erythropoietin and Wnt1 govern pathways of mTOR, Apaf-1, and XIAP in inflammatory microglia. Curr Neurovasc Res 8: 270-285, 2011.

7. Zeng KW, Wang XM, Ko H, Kwon HC, Cha JW and Yang HO: Hyperoside protects primary rat cortical neurons fromneurotoxicity induced by amyloid $\beta$-protein via the PI3K/Akt/Bad/Bcl(XL)regulated mitochondrial apoptotic pathway. Eur J Pharmacol 672: $45-55,2011$
8. Koh PO: Nicotinamide attenuates the ischemic brain injury-induced decrease of Akt activation and Bad phosphorylation. Neurosci Lett 498: 105-109, 2011.

9. Zhou X, Wang L, Wang M, Xu L, Yu L, Fang T and Wu M: Emodin-induced microglial apoptosis is associated with TRB3 induction. Immunopharmacol Immunotoxicol 33: 594-602, 2011.

10. Porcile C, Di Zazzo E, Monaco ML, D'Angelo G, Passarella D, Russo C, Di Costanzo A, Pattarozzi A, Gatti M, Bajetto A, et al: Adiponectin as novel regulator of cell proliferation in human glioblastoma. J Cell Physiol 229: 1444-1454, 2014.

11. Lu C, Wang W, Jia Y, Liu X, Tong Z and Li B: Inhibition of AMPK/autophagy potentiates parthenolide-induced apoptosis in human breast cancer cells. J Cell Biochem 115: 1458-1466, 2014.

12. Lin YY, Chen CY, Chuang TY, Lin Y, Liu HY, Mersmann HJ, Wu SC and Ding ST: Adiponectin receptor 1 regulates bone formation and osteoblast differentiation by GSK-3 $\beta / \beta$-Catenin signaling in mice. Bone 64: 147-154, 2014.

13. Zhang Y, Sun S, Chen J, Ren P, Hu Y, Cao Z, Sun H and Ding Y: Oxymatrine induces mitochondria dependent apoptosis in human osteosarcoma MNNG/HOS cells through inhibition of PI3K/Akt pathway. Tumour Biol 35: 1619-1625, 2014.

14. Vetvik KK, Sonerud T, Lindeberg M, Lüders T, Størkson RH, Jonsdottir K, Frengen E, Pietiläinen KH and Bukholm I: Globular adiponectin and its downstream target genes are up-regulated locally in human colorectal tumors: ex vivo and in vitro studies. Metabolism 63: 672-681, 2014.

15. Bertoldo F, Silvestris F, Ibrahim T, Cognetti F, Generali D, Ripamonti CI, Amadori D, Colleoni MA, Conte P, Del Mastro L, et al: Targeting bone metastatic cancer: Role of the mTOR pathway. Biochim Biophys Acta 1845: 248-254, 2014.

16. Klubo-Gwiezdzinska J, Costello J Jr, Patel A, Bauer A, Jensen K, Mete M, Burman KD, Wartofsky L and Vasko V: Treatment with metformin is associated with higher remission rate in diabetic patients with thyroid cancer. J Clin Endocrinol Metab 98: 3269-3279, 2013

17. Shackelford DB, Abt E, Gerken L, Vasquez DS, Seki A, Leblanc M, Wei L, Fishbein MC, Czernin J, Mischel PS, et al: LKB1 inactivation dictates therapeutic response of non-small cell lung cancer to the metabolism drug phenformin. Cancer Cell 23: 143-158, 2013.

18. Russo GL, Russo M and Ungaro P: AMP-activated protein kinase: a target for old drugs against diabetes and cancer. Biochem Pharmacol 86: 339-350, 2013.

19. Algire C, Amrein L, Bazile M, David S, Zakikhani M and Pollak M: Diet and tumor LKB1 expression interact to determine sensitivity to anti-neoplastic effects of metformin in vivo. Oncogene 30: 1174-1182, 2011.

20. Molosh AI, Johnson PL, Fitz SD, Dimicco JA, Herman JP and Shekhar A: Changes in central sodium and not osmolarity or lactate induce panic-like responses in a model of panic disorder. Neuropsychopharmacology 35: 1333-1347, 2010.

21. Green AS, Chapuis N, Maciel TT, Willems L, Lambert M, Arnoult C, Boyer O, Bardet V, Park S, Foretz M, et al: The LKB1/AMPK signaling pathway has tumor suppressor activity in acute myeloid leukemia through the repression of mTOR-dependent oncogenic mRNA translation. Blood 116: 4262-4273, 2010

22. Roohi A and Hojjat-Farsangi M: Recent advances in targeting mTOR signaling pathway using small molecule inhibitors. J Drug Target 25: 189-201, 2017.

23. Moench R, Grimmig T, Kannen V, Tripathi S, Faber M, Moll EM, Chandraker A, Lissner R, Germer CT, Waaga-Gasser AM, et al: Exclusive inhibition of $\mathrm{PI} 3 \mathrm{~K} / \mathrm{Akt} / \mathrm{mTOR}$ signaling is not sufficient to prevent PDGF-mediated effects on glycolysis and proliferation in colorectal cancer. Oncotarget 7: 68749-68767, 2016. 